It is a useful technique to exclude $H$. pylori gastritis. The clinical relevance is that this technique allows for targeted biopsies, reducing the miss rate and thus increasing the diagnostic yield.

Disclosure of Interest J. White: None Declared, S. Sami: None Declared, J. Ortiz Fernández-Sordo: None Declared, J. Mannath: None Declared, K. Ragunath Grant/research support from: Olympus-Keymed UK, Speaker honoraria and consultancy fees from: Olympus-Keymed UK.

\section{PTU-034 DOUBLE BLIND RANDOMISED CONTROLLED TRIAL OF MAGNETICALLY STEERABLE GASTRIC CAPSULE ENDOSCOPY (MSGCE) VS. CONVENTIONAL GASTROSCOPY FOR DETECTION OF BEADS IN A PORCINE STOMACH}

${ }^{1} \mathrm{MF}$ Hale*, ${ }^{2}$ Rahman, ${ }^{1} \mathrm{~K}$ Drew, ${ }^{1} \mathrm{R}$ Sidhu, ${ }^{3} \mathrm{~S} A$ Riley, ${ }^{2} \mathrm{P}$ Patel, ${ }^{1} \mathrm{ME}$ McAlindon. ${ }^{1}$ Gastroenterology, Royal Hallamshire Hospital, Sheffield, UK; ${ }^{2}$ Gastroenterology, Southampton Hospital University Trust, Southampton, UK; ${ }^{3}$ Gastroenterology, Northern General Hospital, Sheffield, UK

\subsection{6/gutjnl-2014-307263.108}

Introduction Gastroscopy is uncomfortable for patients and incurs the risks of intubation and sedation. Capsule endoscopy is well tolerated and recently a handheld magnet has been developed to enable steering of the capsule to visualise all areas of the capacious stomach. Our preliminary data suggests that a novice can identify all beads sewn into a porcine stomach within 4 min after 40 consecutive examinations. ${ }^{1}$ We performed a double blind randomised controlled trial comparing MSGCE with conventional gastroscopy in the detection of beads in the same model.

Methods Ex-vivo porcine stomach models were used in a standard housing unit. MSGCE was performed according to a standard protocol using $1000 \mathrm{mls}$ of water to distend each stomach and a combination of positional change (head down, $30^{\circ}$ left lateral, $30^{\circ}$ right lateral) and magnetic control to steer the capsule. Each model was examined in a standard fashion by gastroscopy and subsequently MSGCE using MiroCam Navi (Intromedic Ltd). Two blinded investigators (MFH and IR) competent to perform both procedures were allocated randomly to perform either gastroscopy or MSGCE on each model.
This was performed as a non-inferiority study with an expected sensitivity of $90 \%$ for both (0 estimated difference), a specificity of $100 \%$ and a difference of interest 10 percentage points (i.e., $80 \%$ is significantly worse). A sample size of 85 beads was needed to achieve this statistical power. Twelve porcine stomachs were prepared with beads as follows: $2 \times 0$ beads, $2 \times 1$ bead, $2 \times 2$ beads, $2 \times 3$ beads, $2 \times 4$ beads, 2 $\times 5$ beads, giving a total of 30 beads. The study was conducted in three rounds, giving a total of 90 beads to be identified. Number of beads identified and procedure duration was recorded.

Results Gastroscopy correctly identified 88\% (79/90) beads, MSGCE correctly identified $89 \%(80 / 90)$ beads and thus is noninferior to gastroscopy in this setting (95\% CI 82.54-95.46\%). Mean examination times for gastroscopy and MSGCE were 3.34 min and 9.90 min respectively. MSGCE overestimated the number of beads present on a single occasion.

Conclusion MSGCE is equivalent to conventional gastroscopy in the detection of beads placed in a porcine stomach model. Procedure duration was longer for MSGCE compared to gastroscopy. Further studies in humans are necessary to define the scope and utility of this exciting new technique.

\section{REFERENCE}

1 Hale MF, Drew K, Baldacchino T, Anderson S, Sanders DS, Riley SA, Sidhu R, McAlindon ME. Gastroscopy without a gastroscope! Feasibility in a porcine stomach model using a magnetic capsule. Abstract. British Society of Gastroenterology Annual Meeting, Jun 2013

Disclosure of Interest None Declared.

\section{PTU-035 SINGLE CENTRE EXPERIENCE WITH ENDOCLOT POWDER SPRAY FOR UPPER GASTROINTESTINAL BLEED}

M Kasimanickam*, S Vinnamala, MR Andrew, C Lim, M Ahmed. Gastroenterology, Good Hope Hospital, Sutton Coldfield, UK

\subsection{6/gutjnl-2014-307263.109}

Introduction Endoclot' (EPI) and 'Hemospray' (Wilson Cook) are haemostatic powders marketed for endoscopic use. The

\begin{tabular}{|c|c|c|c|c|c|}
\hline Age and sex & Endo diagnosis & Endotherapy & Co morbidity & Outcome & $\begin{array}{l}30 \text { days } \\
\text { mortality } \\
\mathrm{Y} / \mathrm{N}\end{array}$ \\
\hline $63 \mathrm{~F}$ & DU & Adrenaline + balloon tamponade + & DM, stroke, CKD, COAD & Haemostasis & Y \\
\hline $92 \mathrm{~F}$ & DU & Endoclot & Leukaemia, TIA, HT, asthma & Haemostasis; died 11 days later, & $\mathrm{N}$ \\
\hline $85 \mathrm{M}$ & Multiple & Adrenaline + Endoclot (partial) & CVA, COPD, CKD & pneumonia & Y \\
\hline $87 \mathrm{M}$ & DU & Adrenaline + clips + gold probe + & $C V A, C K D, A F, H T$ & Haemostasis & $\mathrm{N}$ \\
\hline $88 \mathrm{M}$ & DU & Endoclot $\times 2$ & $\mathrm{MI}, \mathrm{AF}$ & Died 3 days later due to sepsis & $\mathrm{N}$ \\
\hline \multirow[t]{2}{*}{$83 \mathrm{M}$} & DU & Endoclot & COPD, CVA, AF, HT, CKD, & Died 5 days later, pneumonia & Y \\
\hline & DU & $\begin{array}{l}\text { Adrenaline + Endoclot } \\
\text { Adrenaline + Goldprobe + } \\
\text { Clips + Endoclot }\end{array}$ & Carotid endarterectomy & $\begin{array}{l}\text { Died } 19 \text { days later due to } \\
\text { cardiac failure }\end{array}$ & \\
\hline $63 \mathrm{M}$ & $\begin{array}{l}\text { Bleeding lymphoma - } \\
\text { 4th part of duodenum }\end{array}$ & Endoclot via enteroscope & End stage follicular lymphoma & Died next day & $\mathrm{N}$ \\
\hline $89 \mathrm{~F}$ & GU & Adrenaline + Endoclot & Cholangitis & Haemostasis & Y \\
\hline $67 \mathrm{~F}$ & Severe bleed after gastric polyp biopsy & Adrenaline + Endoclot & $\mathrm{DM}, \mathrm{CKD}, \mathrm{HT}$ & Haemostasis & Y \\
\hline 77M & Gastric Erosions/ Gastric Lymphoma & Adrenaline + Goldprobe + Endoclot & Lymphoma & Died 5 days later, late rebleed & $\mathrm{N}$ \\
\hline $81 \mathrm{M}$ & GOJ Tear Post ERCP & Adrenaline + Endoclot & & Haemostasis & Y \\
\hline $83 \mathrm{M}$ & GIST & Endoclot & $\mathrm{AF}, \mathrm{MI}, \mathrm{CVA}$ & Haemostasis & Y \\
\hline
\end{tabular}

